\title{
The $k$-Yamabe problem
}

\author{
Weimin Sheng, Neil S. Trudinger, and Xu-Jia Wang
}

\section{Introduction}

1.1. The $k$-Yamabe problem. The $k$-Yamabe problem is a higher order extension of the celebrated Yamabe problem for scalar curvature. It was initially proposed by Viaclovsky $[\mathbf{7 2}]$ and also arose in the study of Q-curvatures in [11]. Viaclovsky found that in a conformal metric, the resultant $k$-curvature equation can be expressed as an equation similar to the $k$-Hessian equation, which has been studied by many authors [77].

The $k$-Yamabe problem can be formulated as follows. Let $\left(\mathcal{M}^{n}, g\right)$ be a closed Riemannian manifold of dimension $n \geq 3$. It is well-known that there is an orthonormal decomposition of the Riemannian curvature tensor

$$
\text { Riem }=W+A_{g} \odot g,
$$

where $W$ is the Weyl tensor and $A_{g}$ is the Schouten tensor. The Weyl tensor is conformally invariant and Schouten tensor is given by

$$
A_{g}=\frac{1}{n-2}\left(R i c_{g}-\frac{R_{g}}{2(n-1)} g\right),
$$

where $\operatorname{Ric}_{g}$ and $R_{g}$ are respectively the Ricci tensor and the scalar curvature. For $1 \leq k \leq n$, the $k$-Yamabe problem is to prove the existence of a conformal metric $\widetilde{g}$ such that

$$
\sigma_{k}\left(\lambda\left(A_{\tilde{g}}\right)\right)=1 \text { on } \mathcal{M},
$$

where $\lambda=\left(\lambda_{1}, \ldots, \lambda_{n}\right)$ are the eigenvalues of the Schouten tensor $A_{\widetilde{g}}$ with respect to $\widetilde{g}, \sigma_{k}(\lambda)$ is the $k$-th elementary symmetric polynomial,

2000 Mathematics Subject Classification. 53C21, 58J05, 35J60.

Key words and phrases. Yamabe problem, nonlinear elliptic equation, existence, compactness.

The first author was supported by NSFC 10771189 and 10831008. The second and third authors were supported by the Australian Research Council.

(C)2012 International Press 
given by

$$
\sigma_{k}\left(\lambda_{1}, \ldots, \lambda_{n}\right)=\sum_{1 \leq i_{1}<\cdots<i_{k} \leq n} \lambda_{i_{1}} \lambda_{i_{2}} \cdots \lambda_{i_{k}}
$$

For convenience we call $\sigma_{k}\left(\lambda\left(A_{\tilde{g}}\right)\right)$ the $k$-scalar curvature, or simply $k$-curvature, of $(\mathcal{M}, \widetilde{g})$.

Let

$$
\widetilde{g}=e^{-2 w} g=v^{\frac{4}{n-2}} g, \quad\left(v=e^{-\frac{n-2}{2} w}\right) .
$$

By direct computation,

$$
A_{\widetilde{g}}=\nabla^{2} w+d w \otimes d w-\frac{1}{2}|\nabla w|^{2} g+A_{g} .
$$

Hence the $k$-Yamabe problem is equivalent to solving the following PDE

$$
\sigma_{k}\left[\lambda\left(\nabla^{2} w+d w \otimes d w-\frac{1}{2}|\nabla w|^{2} g+A_{g}\right)\right]=e^{-2 k w} .
$$

When $k \geq 2$, this is a fully nonlinear partial differential equation closely related to the $k$-Hessian equation. Therefore techniques developed in the study of the Yamabe problem and the $k$-Hessian equation may be applied to the $k$-Yamabe problem.

1.2. The Yamabe problem. It is convenient to have a brief review of the classical Yamabe problem and the $k$-Hessian equation. The Yamabe problem, proposed by $\mathrm{H}$. Yamabe in [80], is a higher dimensional extension of the famous uniformization theorem, namely any two dimensional Riemannian maniflod is conformally equivalent to one of constant Gauss curvature. Yamabe's paper [80] contains the following theorem on the existence of a conformal metric with constant scalar curvature.

TheOREM 1.1. Let $(\mathcal{M}, g)$ be a compact Riemannian manifold of dimension $n \geq 3$ without boundary. Then there exists a conformal metric $\tilde{g}=$ $v^{4 /(n-2)} \mathrm{g}$ of constant scalar curvature.

Yamabe's proof is based on the variational approach. By direct computation, $\tilde{g}=v^{4 /(n-2)} g$ is a solution to the Yamabe problem if $v$ satisfies the equation

$$
-\frac{4(n-1)}{n-2} \Delta_{g} v+R_{g} v=v^{\frac{n+2}{n-2}}
$$

or equivalently $v$ is a critical point of the functional

$$
\mathcal{F}_{1}(v)=\frac{\int_{\mathcal{M}}\left(\frac{4(n-1)}{n-2}|\nabla v|_{g}^{2}+R v^{2}\right) d v o l_{g}}{\left(\int_{\mathcal{M}} v^{\frac{2 n}{n-2}} d v o l_{g}\right)^{\frac{n-2}{n}}} .
$$


where $\Delta_{g}$ is the Laplace-Beltrami operator, $R_{g}$ is the scalar curvature of $g$. However, Yamabe's proof is correct only when the Yamabe constant $Y_{1} \leq 0$. His proof contains a gap when $Y_{1}>0$, due to the lack of compactness in the Sobolev embedding $W^{1,2} \rightarrow L^{2 n /(n-2)}$. The second author [63] found the gap and fixed it when $Y_{1}<\varepsilon$ for some constant $\varepsilon$, (depending on the Sobolev constant). In $[\mathbf{1}, \mathbf{2}]$, Aubin proved the above theorem under the condition

$$
Y_{1}(g)<Y_{1}\left(g_{S^{n}}\right),
$$

where $g_{S^{n}}$ denotes the standard metric of the $n$-sphere,

$$
Y_{1}(g)=\inf \left\{\mathcal{F}_{1}(v) \mid v>0, v \in C^{\infty}(\mathcal{M})\right\}
$$

is the Yamabe constant. Aubin also verified (1.8) when $n \geq 6$ and $(\mathcal{M}, g)$ is not locally conformally flat. Schoen $[\mathbf{5 1}]$ verified (1.8) for the remaining cases by the positive mass theorem. The positive mass theorem implies a positive lower order term in the expansion of the fundamental solution, which is the key to verify the inequality $(1.8)$. We refer the reader to $[\mathbf{4 0 , 3 , 5 6 ]}$ for a detailed account.

The resolution of the Yamabe problem was a milestone in differential geometry, and has stimulated great interest in the study of various prescribing curvature problems, including the $k$-Yamabe problem.

An interesting problem, raised by Schoen, is the compactness of the solution set when the manifold is not conformally equivalent to the unit sphere. The compactness problem has drawn much attention in recent years. For the 1-Yamabe problem, this problem has been completely resolved. Schoen first proved the compactness in the case when the manifold is locally conformally flat. He also developed a series of local estimates and fine local analysis for possible blow-ups of solutions to equation $(1.6)[\mathbf{5 2}, \mathbf{5 3}, \mathbf{5 4}, \mathbf{5 7}]$. Some of his estimates can be found in [35]. His estimates were used by many researchers in the study of semilinear elliptic equations, including the compactness problem, and eventually led to the resolution of the compactness of the solution set for dimensions $n=3$ in $[\mathbf{4 7}], n=4,5$ in $[\mathbf{1 7}], n=6,7$ in $[\mathbf{4 5}, \mathbf{4 9}]$, and also the compactness when $n \leq 11$ under the assumption of positive mass theorem in [46]. Recently, Khuri, Marques and Schoen [34] established the compactness for dimensions $n \leq 24$, and Brendle and Marques [6, 7] found the compactness does not hold when the dimension $n \geq 25$.

1.3. The $k$-Hessian equation. When $2 \leq k \leq n$, (1.5) is a fully nonlinear partial differential equation. It is closely related to the $k$-Hessian equation,

$$
\sigma_{k}\left[\lambda\left(D^{2} u\right)\right]=f \quad \text { in } \Omega,
$$

where $\Omega$ is a bounded domain in $\mathbb{R}^{n}, D^{2} u$ is the Hessian matrix of $u$, and $\lambda$ denotes the eigenvalues of $D^{2} u$. When $k=1,(1.10)$ is the Poisson equation, 
when $k=n$ it is the Monge-Ampere equation. For $2 \leq k \leq n$, the $k$-Hessian equation is elliptic when restricted to the space of admissible functions, just like the Monge-Ampere equation when restricted to convex functions.

The $k$-Hessian equation has been studied by many authors. The regularity of $k$-admissible solutions was established by Caffarelli, Nirenberg and Spruck $[\mathbf{1 0}]$. See also Ivochkina $[\mathbf{3 3}]$ for some special cases, and Trudinger [65] for Hessian quotient equations. According to Caffarelli, Nirenberg and Spruck [10], a function is $k$-admissible if the eigenvalues of the Hessian matrix $D^{2} u$ lie in the convex cone

$$
\Gamma_{k}^{+}=\left\{x \in \mathbb{R}^{n} \mid \sigma_{j}(x)>0 \text { for } 1 \leq j \leq k\right\},
$$

and equation (1.10) is elliptic when $u$ is $k$-admissible. For some $k \geq 2, \mathbb{R}^{n}$ contains other cones in which the equation (1.10) is elliptic but in these cases the regularity is unclear at the moment.

The $k$-Hessian equation is of divergence form. A Sobolev type inequality for $k$-admissible functions was proved by Wang [75], and a Moser-Trudinger type inequality was proved by Tian and Wang [61]. The corresponding variational solutions, based on gradient flow approach, were obtained by Chou and Wang [16]. Furthermore, various potential theoretic results have also been obtained for the $k$-Hessian equation by Trudinger and Wang $[\mathbf{6 6}, \mathbf{6 7}, \mathbf{6 8}]$ and Labutin $[\mathbf{3 9}]$, just like those for the Laplacian operator. See also $[\mathbf{7 7}]$ for details.

1.4. Admissibility of metrics. Following Caffarelli, Nirenberg and Spruck [10], we say a metric $\widetilde{g} \in[g] k$-admissible (or simply admissible) if $\lambda\left(A_{\widetilde{g}}\right) \in \Gamma_{k}^{+}$. Hence $\widetilde{g}$ is 1-admissible iff $\sum \lambda_{i}>0$ and $\widetilde{g}$ is $n$-admissibe iff $\lambda_{i}>0$ for all $i$. As in [10], one can show that equation (1.5) is elliptic if the eigenvalues $\lambda\left(A_{\tilde{g}}\right) \in \Gamma_{k}^{+}$. For simplicity we also call the functions $u$ and $w$ $k$-admissible if the metric $\widetilde{g}$ in (1.3) is $k$-admissible. Denote

$$
\begin{aligned}
& {[g]=\left\{\widetilde{g}=v^{4 /(n-2)} g \mid v>0, v \in C^{\infty}(\mathcal{M})\right\},} \\
& {[g]_{k}=\{\widetilde{g} \in[g] \mid \widetilde{g} \text { is } k \text {-admissible }\}}
\end{aligned}
$$

where $[g]$ is the conformal class of $[g]$ and $[g]_{k}$ is the set of $k$-admissible metrics.

Based on the progress in the study of the $k$-Hessian equation, we wish to prove related results for the $k$-Yamabe problem, such as the existence of $k$-admissible solutions to the $k$-Yamabe problem when the initial metric $g$ is $k$-admissible, and related variational properties. However, we would also like to draw the reader's attention to various differences between these two equations. See Remarks 3.1, 4.1 and 4.2.

1.5. Plan of this paper. Assuming the initial metric $g$ is $k$-admissible for some $2 \leq k \leq n$, the $k$-Yamabe problem has been solved in the following 
cases:

- $(\mathcal{M}, g)$ is locally conformally flat, using the moment map of Schoen and Yau;

- $k=2$, by the variational structure of equation (1.5) in this case;

- $k \geq \frac{n}{2}$, in which case, the Ricci curvature is nonnegative.

Resolution of the $k$-Yamabe problem in these cases is built upon the a priori estimates and the Liouville theorem. This paper is arranged as follows.

\section{§2. Liouville theorem}

The Liouville theorem for the case $k=1$ was obtained in [9]. Under certain asymptotic assumptions at infinity, the Liouville theorem was proved in [19] and also follows from $[\mathbf{5 0}]$. A. Li and Y. Li $[\mathbf{4 1}, 42]$ extended the Liouville theorem to the cases $2 \leq k \leq n$. The Liouville theorem was also obtained in [13] for $k=2$ in lower dimensions.

\section{§3. A priori estimates}

By the regularity theory of Evans and Krylov for fully nonlinear, uniformly elliptic equations, it suffices to establish second derivative estimates for solutions of (1.5). If the solution is uniformly bounded, the second derivative estimate can be obtained by differentiating the equation (1.5) twice and applying the maximum principle [73]. But as with the Yamabe problem, a key issue is to establish the uniform estimate. For this purpose, one needs to prove the local gradient and second derivative estimates.

For equation (1.5), the local second derivative estimate can be obtained as in $[\mathbf{2 8}, \mathbf{7 6}]$. Indeed after a proper change of the function $w$, the matrix (1.4) corresponds to that in the equation arising in the reflector design problem. The interior gradient estimate for (1.5) was first obtained by P. Guan and G. Wang [25]. Different proofs were later found in $[\mathbf{1 4}, \mathbf{7 8}, \mathbf{4 4}]$ and they apply to more general equations.

\section{$\S 4$. Existence and compactness}

This section is the main body of the paper. We divide it into four subsections.

§4.1. Existence on locally conformally flat manifolds.

Due to the characterization of locally conformally flat manifolds by Schoen and Yau, and the invariance of the matrix (1.4) under the Kelvin transform, one can apply the method of moving planes to get the uniform estimate. Therefore the existence of solutions can be obtained by either the elliptic method $[\mathbf{4 1}]$ or the parabolic method [26]. The existence of solutions has also been obtained by a variational approach in $[\mathbf{6 0}]$.

\section{§4.2. Existence when the $k$-Yamabe problem is variational.}

It is known that equation (1.5) is variational when $k=2$ or $\mathcal{M}$ is locally conformally flat. In $[60]$ we proved the existence of solutions when equation (1.5) is variational, namely it is the Euler equation of a functional. Our 
result extended the one in $[\mathbf{1 1}, \mathbf{1 2}]$ where the existence was proved when $k=2$ and $n=4$; and that in $[\mathbf{2 6}, \mathbf{4 1}]$ on locally conformally flat manifolds. Note that our proof applies to the cases $k \geq 3$ for any manifolds, provided the equation (1.5) is variational. However, Branson and Gover [5] proved that when $k \geq 3$, equation (1.5) is variational if and only if the manifold $\mathcal{M}$ is locally conformally flat.

§4.3. Existence for $k>n / 2$.

When $k>n / 2$, the existence of solutions was proved by Gursky and Viaclovsky in [30]. They also proved the set of solutions is compact. However in the cases $k>n / 2$, much stronger results were proved by the authors in [70]. It was proved in [70] that the set of admissible metrics, after normalization such that the underlying manifold is of constant volume, is compact. Therefore one can prove the existence of solutions for more general nonlinear right hand side term in (1.5). Some other interesting results on the singularity profile for admissible functions were also established.

$\S 4.4$. Existence for $k=n / 2$.

The cases $k=2$ and $k=\frac{n}{2}$ are probably the most interesting cases for the $k$-Yamabe problem (except $k=1$ ). In the case $k=n / 2$, we proved the solution set is compact and so also the existence of solutions [71]. As in [70], our proof is based on the analysis of a minimal radial function of the solution and also relies on the Liouville theorem of A. Li and Y. Li. A key fact for the cases $k \geq n / 2$ is that if $g$ is $k$-admissible, then the Ricci curvature of the manifolds is nonnegative, so that the Bishop's volume growth theorem is applicable.

For the compactness of the solution set of the $k$-Yamabe problem, when $k<\frac{n}{2}$, nothing has been proved, but we expect similar results as the 1Yamabe problem, namely the solution set is compact in lower dimensions

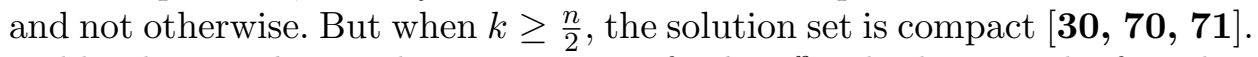
Unlike the case $k=1$, the compactness for $k \geq \frac{n}{2}$ is built upon the fact that a $k$-admissible metric must have nonnegative Ricci curvature $[\mathbf{2 4}, \mathbf{7 1}]$.

$\S 5$. Remarks.

We include in $\S 5.1$ a result proved by the first author in [59], which says that if a $k$-Yamabe constant is positive, then there is a $k$-admissible metric. In $\S 5.2$ we explain that the results obtained for equation (1.5) apply to a larger class of comformally invariant equations satisfying certain structural conditions. Finally we mention some unsolved problems.

\section{Liouville theorem}

Let $\tilde{g}=v^{\frac{4}{n-2}} g$. Then the Schouten tensor of $\tilde{g}$ is given by

$$
A_{\widetilde{g}}=\frac{2}{n-2} \frac{\nabla_{c}^{2} v}{v}+A_{g}
$$


and the $k$-Yamabe problem (1.1) becomes

$$
\sigma_{k}\left(\lambda\left(\nabla_{c}^{2} v+\frac{n-2}{2} v A_{g}\right)\right)=\left(\frac{n-2}{2}\right)^{k} v^{k \frac{n+2}{n-2}} .
$$

where

$$
\nabla_{c}^{2} v=-\nabla^{2} v+\frac{n}{n-2} \frac{d v \otimes d v}{v}-\frac{|\nabla v|^{2}}{v} \frac{g}{n-2}
$$

is the conformal Hessian matrix.

The following Liouville type theorem is due to Caffarelli, Gidas and Spruck [9] (see also [35]) for $k=1$ and by A. Li and Y. Y. Li [41, 42] for $k \geq 2$. The Liouville theorem has been used in the proof for the existence of solutions to the $k$-Yamabe problem for $k=\frac{n}{2}[\mathbf{7 1}]$. It has also been used to establish the local gradient estimate for the equation $(2.1)[\mathbf{4 4}, \mathbf{7 8}]$.

TheOREm 2.1. Let $v \in C^{2}\left(R^{n}\right)$ be a positive, $k$-admissible solution of

$$
\sigma_{k}\left(\lambda\left(\nabla_{c}^{2} v\right)=\left(\frac{n-2}{2}\right)^{k} v^{k \frac{n+2}{n-2}-\varepsilon}\right.
$$

in the entire space $\mathbb{R}^{n}$, where $n \geq 3,1 \leq k \leq n$, and $0 \leq \varepsilon<k \frac{n+2}{n-2}$. Then $\varepsilon=0$ and there exist a constant $a>0$ and a point $\bar{x} \in \mathbb{R}^{n}$ such that

$$
v(x)=c(n, k)\left(\frac{a}{1+a^{2}|x-\bar{x}|^{2}}\right)^{\frac{n-2}{2}},
$$

where $c(n, k)=2^{\frac{n-2}{4}}\left(\begin{array}{l}n \\ k\end{array}\right)^{\frac{n-2}{4 k}}$.

When $k=1$ and $\varepsilon=0$, equation (2.3) becomes

$$
-\Delta v=\frac{n-2}{2} v^{\frac{n+2}{n-2}} \quad \text { on } \mathbb{R}^{n} .
$$

The above Liouville theorem asserts that a positive $C^{2}$ solution of (2.5) must be of the form

$$
v(x)=(2 n)^{\frac{n-2}{4}}\left(\frac{a}{1+a^{2}|x-\bar{x}|^{2}}\right)^{\frac{n-2}{2}} .
$$

Under the decay hypothesis $v(x)=O\left(|x|^{2-n}\right)$, the result was proved in [19] and also follows from Obata [50]. Obata proved the uniqueness of solutions (up to conformal diffeomorphism) for the Yamabe problem on the unit sphere.

Obata's result was extended to $k \geq 2$ by Viaclovsky $[\mathbf{7 2}, \mathbf{7 4}]$. By the stereographic projection, Viaclovsky's uniqueness result implies the following: If $v$ is a $k$-admissible solution to $(2.3)$ and if the function $\tilde{v}(x):=$ $|x|^{2} v\left(\frac{x}{|x|^{2}}\right)$ is smooth and positive at the origin, then $v$ must be given by (2.4). This result can be proved by the method of moving planes as the equation (2.3) is invariant under Kelvin transform [74]. In the case $k=2$ and 
$n=4,5$, Theorem 2.1 was also proved by Chang, Gursky and Yang $[\mathbf{1 2}, \mathbf{1 3}]$ by different argument, where some further results were also obtained.

The proof of Theorem 2.1 by A. Li and Y. Li $[41,42]$ is also based on the Kelvin transform and the moving planes technique. But they need to establish some local estimates, such as a Harnack type inequality of Schoen, and prove an asymptotic behavior of the solution at infinity. Their initial proof $[\mathbf{4 1}]$ used the local gradient estimate in $[\mathbf{2 5}]$ but later they were able to avoid the a priori estimate by an observation on the behavior of isolated singularities. The Liouville theorem was extended to weak entire solutions in $[\mathbf{4 3}]$, which in turn can be used to establish the interior gradient estimate $[\mathbf{7 8}, \mathbf{4 4}]$. A. Li and Y. Li have also extended the Liouville theorem to much more general fully nonlinear equations $[\mathbf{4 2}]$.

\section{Local estimates}

In this section we consider a priori estimates for $k$-admissible solutions to the equation

$$
\sigma_{k}\left[\lambda\left(\nabla^{2} w+d w \otimes d w-\frac{1}{2}|\nabla w|^{2} g+A_{g}\right)\right]=f(x) e^{-2 k w} .
$$

Equation (3.1) is a fully nonlinear partial differential equation, analogous to the $k$-Hessian equation (1.10). Assume that $f>0$ and is $C^{1,1}$ smooth. To obtain the $C^{3, \alpha}$ estimate for solutions to equation (3.1), by the regularity theory of Evans [18] and Krylov [36] it suffices to prove the second derivative estimate, so that the equations become uniformly elliptic.

The second derivative estimate on compact manifolds without boundary can be obtained easily by differentiating equation (3.1) twice and using the maximum principle [73]. For Yamabe type problems such as (3.1), the $L^{\infty}$ estimate is usually a key issue, and we need interior gradient and second derivative estimates.

The interior second derivative estimate for (3.1) can be obtained similarly as in $[\mathbf{7 6}, \mathbf{2 8}]$. Indeed, in the case $k=n$, equation $(3.1)$ on sphere (with a different zero order term in the matrix) also arises in the reflector design $[\mathbf{7 6}, \mathbf{2 8}]$. The interior gradient estimate for (3.1) seems more complicated and was first obtained in [25]. Different proofs for the interior gradient estimate were later found in $[\mathbf{1 4}, \mathbf{7 8}, \mathbf{4 4}]$.

The proof by Guan and Wang [25] of the interior gradient estimate relies on the algebraic structure of the operator $\sigma_{k}$. Chen's proof $[\mathbf{1 4}]$ combines the estimates for gradient and second derivatives in a single argument. The proof in $[\mathbf{7 8}, \mathbf{4 4}]$ is based on the Liouville theorem $[\mathbf{4 3}]$. (The third author would like to point out that before his paper [78] was published in early 2006, he was not aware that Yanyan Li was trying to prove the regularity by his Liouville theorem, as there was no indication of it in the early version of $[43])$. 
As pointed out in [78], Chen's proof is the favorable one. It is simpler than those in $[\mathbf{2 5}, \mathbf{7 8}, \mathbf{4 4}]$. The proofs in $[\mathbf{1 4}, \mathbf{2 5}]$ also apply to corresponding parabolic equations. Below we adopt the proof of Chen [14].

TheOREM 3.1. Let $w \in C^{3}\left(\mathcal{M}^{n}\right)$ be a k-admissible solution to (3.1) in the geodesic ball $B\left(x_{0}, r\right)$. Then there is a constant $C$ such that

$$
\max _{B\left(x_{0}, r / 2\right)}\left(|\nabla w|^{2}+\left|\nabla^{2} w\right|\right) \leq C\left(1+\max _{B\left(x_{0}, r\right)} e^{-2 w}\right) .
$$

Proof. The proof also applies to more general equations of the form

$$
F(W)=f(x, w),
$$

where

$$
W=A_{g}+\nabla^{2} w+d w \otimes d w-\frac{1}{2}|\nabla w|^{2} g .
$$

We assume that $F=F(\lambda(W))$ is a function of the eigenvalues $\lambda$ of the matrix $W$, satisfying the following conditions:

(i) $F(\lambda)$ is symmetric in $\lambda$,

(ii) $F$ is homogeneous of degree 1 ,

(iii) $F$ is concave, and

(iv) $F$ is positive in the cone $\left\{\lambda \in \mathbb{R}^{n} \mid \lambda_{1}>0, \ldots, \lambda_{n}>0\right\}$.

Note that the above conditions imply that $F(\lambda(W))$ is elliptic in $w$. In the proof here we assume

$$
F=\left[\sigma_{k}(\lambda(W))\right]^{1 / k}
$$

First, since $W \in \Gamma_{k}^{+}$, we have $\operatorname{tr}_{g} W>0$. Hence

$$
\operatorname{tr}_{g} A_{g}+\Delta w-\frac{n-2}{2}|\nabla w|^{2}>0 .
$$

Therefore we have

$$
|\nabla w|^{2}<C(\Delta w+1) .
$$

This means $\Delta w$ is bounded from below, and an upper bound of $\Delta w$ implies an upper bound of $|\nabla w|^{2}$. Since $k \geq 2$, we also have

$$
0<\sigma_{2}(W)=\frac{1}{2}\left(\left(\operatorname{tr}_{g} W\right)^{2}-|W|^{2}\right),
$$

i.e.,

$$
\left|\nabla^{2} w\right|^{2} \leq C\left((\Delta w)^{2}+1\right) .
$$

Hence an upper bound of $\Delta w$ implies an upper bound for $\left|\nabla^{2} w\right|^{2}$.

To prove the interior estimates, we introduce the auxiliary function

$$
H=\eta\left(\Delta w+|\nabla w|^{2}\right)=: \eta L
$$


where $0 \leq \eta \leq 1$ is a cutoff function satisfying $\eta=1$ in $B_{r / 2}, \eta=0$ outside $B_{r},|\nabla \eta|<C(\sqrt{\eta} / r)$ and $\left|\nabla^{2} \eta\right|<C / r^{2}$. For simplicity we assume $r=1$. Suppose $x_{0}$ is the maximal point of $H$. We may assume $\Delta w$ is positive at $x_{0}$. Take a local coordinates such that $g_{i j}\left(x_{0}\right)=\delta_{i j}$. Then at $x_{0}$ we have

$$
\begin{aligned}
& 0=\nabla_{j} H=\eta_{j}\left(\Delta w+|\nabla w|^{2}\right)+\eta\left(w_{k k j}+2 w_{k} w_{k j}\right), \\
& 0 \geq \nabla_{i} \nabla_{j} H=\left(\eta_{i j}-\frac{2 \eta_{i} \eta_{j}}{\eta}\right) L+\eta L_{i j} .
\end{aligned}
$$

Hence

$$
\begin{aligned}
0 \geq F^{i j} H_{i j} & =F^{i j}\left(\left(\eta_{i j}-\frac{2 \eta_{i} \eta_{j}}{\eta}\right) L+\eta L_{i j}\right) \\
& \geq-C\left(\Sigma F^{i i}\right) L+\eta F^{i j} L_{i j},
\end{aligned}
$$

where $F^{i j}=\frac{\partial F}{\partial r_{i j}}$ is positive definite. Now we compute

$$
\begin{aligned}
F^{i j} L_{i j} & =F^{i j}\left(w_{k k i j}+2 w_{k i} w_{k j}+2 w_{k} w_{k i j}\right) \\
& \geq F^{i j} w_{i j k k}+2 F^{i j}\left(w_{k i} w_{k j}+w_{k} w_{i j k}\right)-C\left(\Sigma F^{i i}\right)\left(1+\left|\nabla^{2} w\right|^{3 / 2}\right) \\
& =I+I I-C\left(\Sigma F^{i i}\right)\left(1+\left|\nabla^{2} w\right|^{3 / 2}\right) .
\end{aligned}
$$

Notice that

$$
W_{i j, k k}=w_{i j k k}+2\left(w_{i k} w_{j k}+w_{i k k} w_{j}\right)-\left(\left|\nabla^{2} w\right|^{2}+w_{l} w_{l k k}\right) \delta_{i j}+A_{i j, k k} .
$$

We have by (3.5)

$$
\begin{aligned}
I= & F^{i j} w_{i j k k} \\
= & F^{i j}\left(W_{i j, k k}-2\left(w_{i k} w_{j k}+w_{i k k} w_{j}\right)+\left(\left|\nabla^{2} w\right|^{2}+w_{l} w_{l k k}\right) \delta_{i j}-A_{i j, k k}\right) \\
\geq & F^{i j} W_{i j, k k}-2 F^{i j} w_{i k} w_{j k}+2 F^{i j} w_{j}\left(\frac{\eta_{i}}{\eta} L+2 w_{k} w_{k i}\right) \\
& +\left(\Sigma F^{i i}\right)\left(\left|\nabla^{2} w\right|^{2}-w_{l}\left(\frac{\eta_{l}}{\eta} L+2 w_{k} w_{k l}\right)\right)-C\left(\Sigma F^{i i}\right)\left(1+\left|\nabla^{2} w\right|^{3 / 2}\right) \\
\geq & F^{i j} W_{i j, k k}+F^{i j}\left(4 w_{k} w_{j} w_{k i}-2 w_{i k} w_{j k}+\left|\nabla^{2} w\right|^{2} \delta_{i j}-2 w_{k} w_{l} w_{k l} \delta_{i j}\right) \\
& -C \eta^{-1 / 2}\left(\Sigma F^{i i}\right)|\nabla w| L-C\left(\Sigma F^{i i}\right)\left(1+\left|\nabla^{2} w\right|^{3 / 2}\right) .
\end{aligned}
$$

Since

we have

$$
W_{i j, k}=w_{i j k}+w_{i k} w_{j}+w_{i} w_{j k}-w_{l k} w_{l} \delta_{i j}+A_{i j, k}
$$

$$
\begin{aligned}
I I= & 2 F^{i j} w_{k i} w_{k j}+2 w_{k} F^{i j}\left(W_{i j, k}-w_{i k} w_{j}-w_{i} w_{j k}+w_{l k} w_{l} \delta_{i j}-A_{i j, k}\right) \\
= & 2 w_{k} F^{i j} W_{i j, k}+F^{i j}\left(2 w_{k i} w_{k j}-4 w_{i} w_{k} w_{k j}+2 w_{k} w_{l k} w_{l} \delta_{i j}\right) \\
& -C\left(\Sigma F^{i i}\right)\left(1+\left|\nabla^{2} w\right|^{3 / 2}\right) .
\end{aligned}
$$


Substituting $I$ and $I I$ into (3.7) we have

$$
\begin{aligned}
F^{i j} L_{i j} \geq & F^{i j} W_{i j, k k}+2 w_{k} F^{i j} W_{i j, k}+\left(\Sigma F^{i i}\right)\left|\nabla^{2} w\right|^{2} \\
& -C \eta^{-1 / 2}\left(\Sigma F^{i i}\right)|\nabla w| L-C\left(\Sigma F^{i i}\right)\left(1+\left|\nabla^{2} w\right|^{3 / 2}\right) .
\end{aligned}
$$

Hence from (3.6) we get

$$
\begin{aligned}
0 \geq \eta F^{i j} H_{i j} \geq & \eta^{2} F^{i j} W_{i j, k k}+2 \eta^{2} w_{k} F^{i j} W_{i j, k}+\eta^{2}\left(\Sigma F^{i i}\right)\left|\nabla^{2} w\right|^{2} \\
& -C\left(\Sigma F^{i i}\right)\left(1+\left(\eta\left|\nabla^{2} w\right|\right)+\left(\eta\left|\nabla^{2} w\right|\right)^{3 / 2}\right) .
\end{aligned}
$$

By (3.3) and (3.4),

$$
C^{-1} L \leq\left|\nabla^{2} w\right| \leq C(L+1)
$$

for some constant $C$. Hence by (3.8),

$$
\begin{aligned}
0 \geq & \eta^{2} F^{i j} W_{i j, k k}+2 \eta^{2} w_{k} F^{i j} W_{i j, k}+(\eta / C)^{2}\left(\Sigma F^{i i}\right) L^{2} \\
& -C\left(\Sigma F^{i i}\right)\left(1+\eta L+(\eta L)^{3 / 2}\right) .
\end{aligned}
$$

By the concavity of $F$,

$$
\begin{aligned}
\Delta F(W) & =F^{i j, r s} W_{i j, k} W_{r s, k}+F^{i j} W_{i j, k k} \\
& \leq F^{i j} W_{i j, k k} .
\end{aligned}
$$

Hence by equation (3.1),

$$
\begin{aligned}
F^{i j} W_{i j, k k} & \geq \Delta\left[f(x) e^{-2 w}\right] \\
& \geq-C\left(\Sigma F^{i i}\right)\left(1+e^{-2 w}\right) L .
\end{aligned}
$$

Here we used the property $\left(\Sigma F^{i i}\right) \geq 1$. Similarly from equation (3.1) we have

$$
\begin{aligned}
w_{k} F^{i j} W_{i j, k} & =\nabla_{k} F(W) w_{k} \\
& =\nabla_{k}\left(f(x) e^{-2 w}\right) w_{k} \\
& \geq-C\left(\Sigma F^{i i}\right)\left(1+e^{-2 w}\right) L .
\end{aligned}
$$

Inserting (3.11) and (3.12) into (3.10), we get

$$
H \leq C\left(1+\max _{B_{r}} e^{-2 w}\right)
$$

for $x \in B_{r / 2}$.

Once the second derivative is bounded, equation (3.1) becomes uniformly elliptic, and Evans-Krylov's regularity theory implies higher order regularity for equation (3.1). 
REMARK 3.1.

(i) Equation (3.1) is also elliptic when the eigenvalues

$$
\lambda\left(A_{\widetilde{g}}\right) \in-\Gamma_{k}^{+} .
$$

If $w$ is a solution to (3.1) with eigenvalues $\lambda\left(A_{\tilde{g}}\right) \in-\Gamma_{k}^{+}$, then $\tilde{w}=-w$ satisfies the equation

$$
\sigma_{k}\left[\lambda\left(\nabla^{2} \tilde{w}-d \tilde{w} \otimes d \tilde{w}+\frac{1}{2}|\nabla \tilde{w}|^{2} g-A_{g}\right)\right]=e^{2 k \tilde{w}} .
$$

However there is no second derivative estimate for solutions to (3.14), a counterexample was given in [60]. See also the HeinzLewy counter-example in $[\mathbf{5 8}]$, and $[\mathbf{7 6}, \mathbf{4 8}]$. The difference is due to the sign of the first derivative term $|\nabla w|^{2}$ in the matrices in (3.1) and (3.14).

(ii) For the $k$-Hessian equation (1.10), regularity for solutions $u$ with eigenvalues $\lambda\left(D^{2} u\right) \in \Gamma_{k}$ also hold for solutions with $\lambda\left(D^{2} u\right) \in$ $\left(-\Gamma_{k}\right)$. But the pure interior second derivative estimate $(3.2)$ does not hold for equation (1.10). When the solution vanishes on the boundary, a Pogorelov type estimate was proved in $[\mathbf{1 6}]$.

(iii) In addition to the convex cones $\Gamma_{k}$ and $-\Gamma_{k}$, equations (1.10) and (3.1) may have other elliptic branches, depending on $k$ and $n$. Namely there are (nonconvex) cones $\Gamma \subset \mathbb{R}^{n}$ such that equation (1.10) (and also (3.1)) is elliptic if the corresponding eigenvalues $\lambda\left(D^{2} u\right) \in \Gamma$. In this case, the existence and continuity of viscosity solutions have been proved by Harvey and Lawson [31], but the regularity is unclear at the moment.

\section{Existence and compactness}

In this section we always assume

(a) $(\mathcal{M}, g)$ is a compact Riemannian manifold of dimension $n \geq 3$, not conformally equivalent to the unit sphere;

(b) the metric $g$ is $k$-admissible, or equivalently the class $[g]_{k}$ is not empty.

As mentioned in the introduction, the existence of solutions to the $k$-Yamabe problem (1.1) for $k \geq 2$ has been proved for

(i) locally conformally flat manifolds $[\mathbf{2 6}, \mathbf{4 1}, \mathbf{6 0}]$;

(ii) the case $k=2[\mathbf{6 0}]$;

(iii) the case $k>n / 2[\mathbf{3 0}, \mathbf{7 0}]$; and

(iv) the case $k=n / 2[\mathbf{7 1}]$.

In the following we address the above four cases separately. 
4.1. Existence on locally conformally flat manifolds. The existence of solutions to the $k$-Yamabe problem on locally conformally flat manifolds in $[\mathbf{2 6}, \mathbf{4 1}]$ is based on the characterization of locally conformally flat manifolds by Schoen and Yau [55], and that in [60] relies on the variational structure of the equation (2.1).

When $\mathcal{M}$ is locally conformally flat, there is a conformal immersion of the universal covering of $\mathcal{M}$ into the unit sphere $S^{n}$. By the stereographic projection, the solution $v$ satisfies equation (2.1) in a domain $\Omega$ in the Euclidean space $\mathbb{R}^{n}$. Moreover, if $\Omega \neq \mathbb{R}^{n}$, then $v(x) \rightarrow+\infty$ as $x \rightarrow \partial \Omega$ [55]. Therefore by the moving plane argument, $v$ is locally uniformly bounded in $\Omega$, which in turn implies that $v$ is uniformly bounded on the manifold $\mathcal{M}$. Once the solution is proven uniformly bounded, the solution can be obtained by a degree argument $[\mathbf{4 1}]$. Instead of the degree argument, the existence can also be obtained by a parabolic equation approach [26], just as in [81] for the case $k=1$. Note that once the solutions are proven uniformly bounded, then the solution set is compact.

4.2. Existence when the $k$-Yamabe problem is variational. It was verified that the $k$-Yamabe problem is variational when $k=2$ or the manifold $\mathcal{M}$ is locally conformally flat $[\mathbf{7 2}, \mathbf{8 ,} 60]$. In $[\mathbf{6 0}]$ we proved the existence of solutions to the $k$-Yamabe problem when equation (2.1) is variational. When $k=2$ and $n=4$, the existence was previously obtained in [12]. As with the 1-Yamabe problem, our proof consists of two steps. The first one is to show that the Yamabe problem is solvable if a Yamabe constant satisfies an inequality like (1.8). The second step is to verify the inequality for manifolds not conformally equivalent to the unit sphere $S^{n}$.

Proposition 4.1. ([72, 8, 60] $)$ The $k$-Yamabe problem is variational if $k=2$ or if the manifold $\mathcal{M}$ is locally conformally flat.

A more general result can be found in [60]. Indeed, an operator $F[v]=$ $F\left[\nabla^{2} v, \nabla v, v, x\right]$ is variational if and only if its linearized operator is selfadjoint. If $F$ is not homogeneous of degree -1 , the functional is given by

$$
\mathcal{F}[v]=\int_{\mathcal{M}} G[v]
$$

where

$$
G[v]=\int_{0}^{1} v F[t v] .
$$

When $F$ is homogeneous of degree -1 , such as the case $k=\frac{n}{2}$ in (4.1), we may consider $v$ as a composite function $v=\phi(w)$ and consider the operator $\widetilde{F}[w]=\phi^{\prime}(w) F(\phi(w))$. Hence the corresponding functional is given by

$$
\mathcal{F}_{n / 2}(w)=\int_{\mathcal{M}} \int_{0}^{1} w F[t w] .
$$


In particular if $v=e^{-\frac{n-2}{2} w}$, then we obtain the functional in [8]

$$
\mathcal{F}_{n / 2}(w)=-\int_{\mathcal{M}} \int_{0}^{1} w \sigma_{n / 2}\left(\lambda\left(A_{g_{t}}\right)\right)
$$

where $g_{t}=e^{-2 t w} g_{0}$.

When $k=2$ and $n=4$, by the Gauss-Bonnet-Chern formula,

$$
8 \pi^{2} \chi(\mathcal{M})=\int_{\mathcal{M}}|W|_{g}^{2} d V o l_{g}+\mathcal{F}_{2}(g) .
$$

The Weyl tensor is invariant under conformal change of metrics. Hence $\mathcal{F}_{2}(g)$ is a conformal invariant [11]. For $k=n / 2$ and $n>4$, it is shown in [72] that $\mathcal{F}_{n / 2}$ is also a constant in $[g]$ when $\mathcal{M}$ is locally conformally flat.

Let $\left(\mathcal{M}, g_{0}\right)$ be a Riemannian manifold. If $g=v^{\frac{4}{n-2}} g_{0}$ is a solution of the $k$-Yamabe problem (1.1), then the Schouten tensor is given by $A_{g}=\frac{2}{(n-2) v} V$, and $v$ satisfies the equation

$$
L[v]:=v^{(1-k) \frac{n+2}{n-2}} \sigma_{k}(\lambda(V))=v^{\frac{n+2}{n-2}},
$$

where

$$
V=-\nabla^{2} v+\frac{n}{n-2} \frac{\nabla v \otimes \nabla v}{v}-\frac{1}{n-2} \frac{|\nabla v|^{2}}{v} g_{0}+\frac{n-2}{2} v A_{g_{0}} .
$$

As with the Yamabe problem, we introduce the $k$-Yamabe constant for $2 \leq$ $k \leq \frac{n}{2}$

$$
Y_{k}(\mathcal{M})=\inf \left\{\mathcal{F}_{k}(g) \mid g \in\left[g_{0}\right]_{k}, \operatorname{Vol}(\mathcal{M}, g)=1\right\}
$$

where

$$
\begin{aligned}
\mathcal{F}_{k}(g) & =\int_{\mathcal{M}} \sigma_{k}\left(\lambda\left(A_{g}\right)\right) d \operatorname{vol}_{g} \\
& =\int_{\mathcal{M}} v^{\frac{2 n}{n-2}-k \frac{n+2}{n-2}} \sigma_{k}(\lambda(V)) d \operatorname{vol}_{g_{0}} .
\end{aligned}
$$

Note that we have ignored a coefficient $\left(\frac{2}{n-2}\right)^{k}$ in the second equality. We have the following.

Theorem 4.1. ([60]) Assume $2 \leq k \leq \frac{n}{2}$, the set $\left[g_{0}\right] \neq \emptyset$, and the operator $L$ is variational. Then there is a $k$-admissible solution to the $k$ Yamabe problem (1.1).

REMARK 4.1.

(i) The condition $\left[g_{0}\right] \neq \emptyset$ is essential for the $k$-Yamabe problem. By this condition we can work in the class of $k$-admissible functions, such that the equation (4.1) is elliptic. 
(ii) When a solution is $k$-admissible, we have the regularity of solutions established in Section 3. When the eigenvalues lie in the negative cone $-\Gamma_{k}$, there is no interior regularity and no existence is known.

(iii) A surprising result, proved by Branson and Gover [5], is that equation (4.1) is variational if and only if $k=2$ or $\mathcal{M}$ is locally conformally flat. So if $k \geq 3$, then (4.1) is variational only if $\mathcal{M}$ is locally conformally flat.

Our proof follows the strategy of Aubin, which consists of two steps. In the first step (Lemma 4.1) we show that if $Y_{k}(\mathcal{M})<Y_{k}\left(S^{n}\right)$, then there is a solution to (4.1). In the second step we verify the inequality $Y_{k}(\mathcal{M})<Y_{k}\left(S^{n}\right)$ (Lemma 4.2).

In the first step one needs to use a gradient flow. One should be cautious with parabolic equations of the form

$$
u_{t}=F[u]+\phi\left[u, \int g\left(D^{2} u\right)\right]
$$

where $F$ is an elliptic operator, $g$ is a function of $D^{2} u, \phi$ is a given function. Under proper conditions one can establish the estimate $\left|\partial_{t} u\right|+\left|\partial_{x}^{2} u\right| \leq C$. The estimate implies that the function $\phi\left[u, \int g\left(D^{2} u\right)\right]$ is bounded and measurable in $t$, it does not mean $\phi\left[u, \int g\left(D^{2} u\right)\right]$ is Lipschitz or Hölder continuous in $t$. Hence one cannot conclude $\|u\|_{C^{2,1}} \leq C$ by Krylov's regularity theory. In the general case the higher regularity was recently obtained in [62].

Lemma 4.1. Under the assumptions of Theorem 4.2, if

$$
Y_{k}(\mathcal{M})<Y_{k}\left(S^{n}\right)
$$

then there is a $k$-admissible solution to (4.1).

Proof. A solution of the $k$-Yamabe problem is a min-max type critical point of the corresponding functional. As we need to restrict ourselves to $k$-admissible functions, we cannot directly use variational theory (such as the Ekeland variational principle). Instead we study a descent gradient flow of the functional and investigate its convergence. We need to choose a special gradient flow for which the a priori estimates can be established.

As with the original Yamabe paper [80], we first study the approximating problems

$$
L(v)=v^{p},
$$

where $1<p \leq \frac{n+2}{n-2}$. (4.6) is the Euler equation of the functional $J_{p}(v)=$ $J_{p}(v ; \mathcal{M})$,

$$
J_{p}(v)=\frac{n-2}{2 n-4 k} \int_{\left(\mathcal{M}, g_{0}\right)} v^{\frac{2 n}{n-2}-k \frac{n+2}{n-2}} \sigma_{k}(\lambda(V))-\frac{1}{p+1} \int_{\left(\mathcal{M}, g_{0}\right)} v^{p+1} .
$$


Let $\phi_{1}=\varepsilon$ and $\phi_{2}=\varepsilon^{-1}$, where $\varepsilon>0$ is a small constant. Then when $k<\frac{n}{2}, J_{p}\left(\phi_{1}\right) \rightarrow 0$ and $J_{p}\left(\phi_{2}\right) \rightarrow-\infty$ as $\varepsilon \rightarrow 0$. Let $P$ denote the set of paths in $\Phi_{k}$ connecting $\phi_{1}$ and $\phi_{2}$, namely

$$
P=\left\{\gamma \in C\left([0,1], \Phi_{k}\right) \mid \gamma(0)=\phi_{1}, \gamma(1)=\phi_{2}\right\}
$$

where $\Phi_{k}$ denote the set of $k$-admissible functions. Denote

$$
c_{p}[\mathcal{M}]=\inf _{\gamma \in P} \sup _{s \in[0,1]} J_{p}(\gamma(s) ; \mathcal{M}) .
$$

Then (4.5) is equivalent to

$$
c_{p}[\mathcal{M}]<c_{p}\left[S^{n}\right]
$$

with $p=\frac{n+2}{n-2}$. By (4.10) we can prove that $J_{p}$ has a min-max critical point $v_{p}$ with $J_{p}\left(v_{p}\right)=c_{p}[\mathcal{M}]$, in the sub-critical case $p<\frac{n+2}{n-2}$. By a blow-up argument, we can also prove that $v_{p}$ converges to a solution of (4.1) under the assumption (4.10).

As in (1.3), let

$$
w=-\frac{2}{n-2} \log v
$$

Our gradient flow is then given by

$$
F[w]-w_{t}=\mu(f(x, w)),
$$

where

$$
F[w]:=\mu\left(\sigma_{k}\left(\lambda\left(A_{g}\right)\right)\right)
$$

and $g=e^{-2 w} g_{0}$. When $f(x, w)=e^{-2 k w}$, a stationary solution of (4.12) is a solution to the $k$-Yamabe problem. We choose

$$
\mu(t)= \begin{cases}t^{1 / k} & t \geq 10 \\ \log t & t \in\left(0, \frac{1}{10}\right)\end{cases}
$$

and

$$
(t-s)(\mu(t)-\mu(s)) \geq c_{0}(t-s)\left(t^{1 / k}-s^{1 / k}\right)
$$

for some constant $c_{0}>0$ independent of $t$. We also assume that $\mu$ is monotone increasing and satisfies

$$
\lim _{t \rightarrow 0^{+}} \mu(t)=-\infty
$$

This condition ensures the solution is $k$-admissible at any time $t$.

We say a solution $w$ to the parabolic equation (4.12) is $k$-admissible if for any fixed $t, w$ is $k$-admissible as a function of $x$. Denote $Q_{r}=B_{r}(0) \times\left(0, r^{2}\right]$. 
To prove Lemma 4.1, we establish the following interior a prori estimates for $w[\mathbf{6 0}]$ :

$$
\begin{aligned}
\left|\nabla_{x} w\left(0, r^{2}\right)\right| & \leq C_{1}, \\
\left|\nabla_{x}^{2} w\left(0, r^{2}\right)\right| & \leq C_{2}, \\
\left|w_{t}\left(0, r^{2}\right)\right| & \leq C_{3},
\end{aligned}
$$

where $C_{1}$ is independent of $\sup w$ (if $f=\kappa(x) e^{-p w}$ for some constant $p>0$ and smooth, positive function $\kappa), C_{2}$ and $C_{3}$ depend only on $n, k, r, \mu, \inf w$, $\sup w$, and $\left\|A_{g_{0}}\right\|_{C^{2}}$.

Estimates (4.13)-(4.15) can be established similarly as for the elliptic equation (3.1), using the arguments in [25] or [14]. For the parabolic equation (4.12), to prove the $L^{\infty}$ estimate we use a blow-up argument and we need to show the estimates (4.13) and (4.14) also hold for the equation

$$
\frac{1}{a} \mu\left(a^{k} \sigma(\lambda(W))\right)-w_{t}=\frac{1}{a} \mu\left(a^{k} f\right),
$$

where $a>0$ is a constant, and the constants $C_{1}, C_{2}$ are independent of $a$ if $a \geq 1$.

By estimates (4.13)-(4.15), equation (4.12) becomes locally uniformly parabolic. By Krylov's regularity theory, we obtain the $C^{4,2}$ a priori estimate and also the local existence. To prove Lemma 4.1, we first consider the existence of $k$-admissible solutions for $2 \leq k<n / 2$ to equation (4.6) in the subcritical growth case $1<p<\frac{n+2}{n-2}$. As a solution to (4.6) is a critical point of the functional $J=J_{p}$, we write the parabolic equation (4.12) in terms of $v$ as

$$
F[v]+\frac{v_{t}}{v}=\mu(f(v))
$$

where $f(v)=v^{\frac{4 k}{n-2}-\varepsilon}, F[v]=\mu\left(\sigma_{k}\left(\lambda\left(\frac{V}{v}\right)\right)\right)$, and $\varepsilon=\frac{n+2}{n-2}-p$. Equation (4.16) is a descent gradient flow of the functional $J(v)$. By a blow-up argument and the Liouville Theorem 2.1, we can prove that there is an initial function such that the solution to (4.16) is uniformly bounded and converges to a solution $v_{p}$ of (4.6) with

$$
J_{p}\left(v_{p}\right)=c_{p}>0,
$$

where $c_{p}$ is the min-max critical value given in (4.9). Moreover, the set of solutions of (4.6) is compact.

Let $p \nearrow \frac{n+2}{n-2}$. By condition (4.5), the Liouville theorem 2.1, and (4.17), we can prove that the solution $v_{p}$ is uniformly bounded. Hence by the a priori estimates, $v_{p}$ converges smoothly along a subsequence to a solution of (4.1). Hence Lemma 4.1 is proved for $2 \leq k<\frac{n}{2}$.

For the case $k=\frac{n}{2}$, we can verify that the functional $\mathcal{F}_{n / 2}(g)$ is a constant, and by (4.5), the constant is strictly smaller than $Y_{n / 2}\left(S^{n}\right)$. Hence 
by the Liouville theorem in $[\mathbf{4 1}]$, one can prove that the set of solutions of (4.1) is compact. The existence of the solutions can be obtained by a degree argument.

For the existence of min-max critical points of fully nonlinear elliptic functionals, a logarithm gradient flow was first used by Chou [15]. More general gradient flows were later used in $[\mathbf{7 5}]$ to prove the Sobolev type inequality (4.20) below and in [16] to prove the existence of min-max critical points of the functional corresponding to the $k$-Hessian equation (1.10). Our argument above was inspired by the argument in $[\mathbf{1 5}, \mathbf{7 5}, \mathbf{1 6}]$.

LEMma 4.2. The critical inequality (4.5) holds for any compact manifold not conformally equivalent to the unit sphere $S^{n}$.

Proof. We reduce the proof of the lemma for $k \geq 2$ to the case $k=1$, namely (1.8). Let $v_{0}$ be the solution to the classic Yamabe equation

$$
-\Delta v+\frac{n-2}{4(n-1)} R v=n(n-2) v^{\frac{n+2}{n-2}} .
$$

Let $v_{k}$ be the solution of

$$
\sigma_{k}(\lambda(V))=C_{n, k} v_{0}^{k \frac{n+2}{n-2}} \text { on } \mathcal{M}
$$

where $C_{n, k}=\frac{n !(n-2)^{k}}{k !(n-k) !}$. Then

$$
-\Delta v_{k}+\frac{n-2}{4(n-1)} R v_{k} \geq n(n-2) v_{0}^{\frac{n+2}{n-2}}
$$

By comparison principle, $v_{k} \geq v_{0}$. By direct computation, $g=v_{k}^{4 /(n-2)} g_{0}$ satisfies

$$
Y_{k}(\mathcal{M}) \leq \mathcal{F}_{k}(g)<Y_{k}\left(S^{n}\right)
$$

This finishes the proof of Lemma 4.2.

As a consequence of our argument, we have the following Sobolev type inequality. For locally conformally flat manifolds, it was first proved in [27].

Corollary 4.1. Let $2 \leq k<\frac{n}{2}$. Then there exists a constant $C>0$ such that the inequality

$$
\left(\operatorname{Vol}\left(\mathcal{M}_{g}\right)\right)^{\frac{n-2}{2 n}} \leq C\left[\int_{\mathcal{M}} \sigma_{k}\left(\lambda\left(A_{g}\right)\right) d \operatorname{vol}_{g}\right]^{\frac{n-2}{2 n-4 k}}
$$

holds for any conformal metric $g=v^{\frac{4}{n-2}} g_{0} \in\left[g_{0}\right]_{k}$.

REMARK 4.2. Inequality (4.18) can be written in the form

$$
\|v\|_{L^{2 n /(n-2)}(\mathcal{M})} \leq C\left[\mathcal{F}_{k}[v]\right]^{\frac{n-2}{2 n-4 k}} .
$$


where $\mathcal{F}_{k}$ is given in (4.4). When $k=1$, we recover the Sobolev inequality

$$
\|v\|_{L^{2 n /(n-2)}(\mathcal{M})} \leq C\left[\int|D v|^{2}\right]^{1 / 2} .
$$

In [27], Guan and Wang also proved the following inequality for locally conformally flat manifolds,

$$
\left[\int_{\mathcal{M}} \sigma_{k}\left(\lambda\left(A_{g}\right)\right) d v l_{g}\right]^{\frac{n-2}{2 n-4 k}} \leq\left[\int_{\mathcal{M}} \sigma_{m}\left(\lambda\left(A_{g}\right)\right) d \operatorname{vol}_{g}\right]^{\frac{n-2}{2 n-4 m}}
$$

for $1 \leq k<m \leq \frac{n}{2}$ (the opposite inequality was proved when $\frac{n}{2} \leq k<m \leq n$ in $[\mathbf{2 7}])$.

The above inequalities are similar to those for the $k$-Hessian equation, proved in $[\mathbf{7 5}, \mathbf{6 9}]$. Let $\Omega$ be a bounded, $(k-1)$-convex domain in $\mathbb{R}^{n}$, i.e. for any point $x \in \partial \Omega, \sigma_{k-1}(\kappa(x))>0$, where $\kappa(x)=\left(\kappa_{1}(x), \ldots, \kappa_{n-1}(x)\right)$ are the principal curvatures of $\partial \Omega$ at $x$. Denote by $\Phi_{0}^{k}(\Omega)$ the set of all $k$-admissible functions vanishing on $\partial \Omega$ and

$$
\|u\|_{\Phi_{0}^{k}}=\left[\int_{\Omega}(-u) \sigma_{k}\left[\lambda\left(D^{2} u\right)\right] d x\right]^{\frac{1}{k+1}}
$$

One easily verifies that $\|\cdot\|_{\Phi_{0}^{k}}$ is a norm in $\Phi_{0}^{k}$. The Sobolev type inequality

$$
\|u\|_{L^{p}(\Omega)} \leq C\|u\|_{\Phi_{0}^{k}}
$$

was proved in [75], where $u \in \Phi_{0}^{k}(\Omega), p \leq k^{*}:=\frac{n(k+1)}{n-2 k}$ and $C=C(n, k$, $p,|\Omega|)$ if $1 \leq k<\frac{n}{2} ; p<\infty$ and $C=C(n, p, \operatorname{diam}(\Omega))$ if $k=\frac{n}{2}$; and $p=\infty$ and $C=C(n, k, \operatorname{diam}(\Omega))$ if $\frac{n}{2}<k \leq n$. In [69] the following inequality was proved,

$$
\|u\|_{\Phi_{0}^{k}} \leq C\|u\|_{\Phi_{0}^{m}}
$$

where $u \in \Phi_{0}^{m}(\Omega), 1 \leq k<m \leq n$. See also [77] for details.

If $1 \leq k<\frac{n}{2}$, the exponent $k^{*}$ in (4.20) is optimal. In [75] it was proved that the best constant in (4.20) is achieved by radially symmetric functions. Therefore when $p=k^{*}$ and $k<\frac{n}{2}$, by the classical Sobolev embedding, the best constant $C$ is attained when $\Omega=\mathbb{R}^{n}$ by the function

$$
u(x)=\left[1+|x|^{2}\right]^{(2 k-n) / 2 k} .
$$

When $k=\frac{n}{2}$, a Moser-Trudinger type inequality has also been proved in [61]. The reader should note the different powers in inequalities (4.19) and (4.20).

In the case $\frac{n}{2}<k \leq n,(4.20)$ can be strengthened. It was shown [67] that a $k$-admissible function to the $k$-Hessian equation (1.10) is Hölder continuous with exponent $\alpha=2-\frac{n}{k}$. This observation gives a guidance for us to prove the results in the next two sub-sections. 
4.3. The cases for $k>n / 2$. The existence of solutions and the compactness of the solution set for the $k$-Yamabe problem have been proved in $[\mathbf{3 0}]$ for $k>n / 2$ and $[\mathbf{7 0 , 7 1 ]}$ for $k \geq n / 2$. In the case $k>n / 2$, much stronger results were proved in [70], i.e., not only the solution set, but the set of all $k$-admissible metrics with fixed volume, is compact. These results are based on the following observation.

Proposition 4.2. If the metric $g$ is $k$-admissible for some $k \geq \frac{n}{2}$, then the Ricci curvature is positive if $k>\frac{n}{2}$ and nonnegative if $k=\frac{n}{2}$.

Proposition 4.2 was first observed in the case $k=2, n=4$ in [11] and proved in the general case in [24]. Our proof of Proposition 4.2 is based on a simple inequality in [67]. Note that the Ricci curvature $\mu=\left(\mu_{1}, \ldots, \mu_{n}\right)$ is given by

$$
\mu_{i}=(n-2) \lambda_{i}+\sum \lambda_{j}
$$

Hence the Ricci curvature $\mu_{i} \geq 0$ for all $1 \leq i \leq n$ if and only if $P_{\frac{1}{n-2}}(\lambda) \geq 0$, where

$$
P_{\delta}(\lambda)=\min \lambda_{i}+\delta \sum \lambda_{i}
$$

is the Pucci operator. For any $\lambda \in \Gamma_{k}$, let $f(x)=\frac{1}{2} \sum \lambda_{k} x_{k}^{2}$. By direct computation, $\Delta_{p} f \geq 0$ for $p \leq 2+\frac{n(k-1)}{n-k}$ (this is the case $l=1$ in Lemma $4.2[\mathbf{6 7}])$. Hence

$$
\sum \lambda_{i}+\frac{n(k-1)}{n-k} \lambda_{j} \geq 0
$$

for every $j$. When $k=\frac{n}{2}, \frac{n(k-1)}{n-k}=n-2$. Hence Ric $_{g} \geq 0$ if $\lambda\left(A_{g}\right) \in \Gamma_{n / 2}$, and Ric $_{g}>0$ if $\lambda\left(A_{g}\right) \in \Gamma_{k}$ for $k>\frac{n}{2}$.

By Proposition 4.2, one can consider the existence of conformal metrics such that the $k$-curvature is equal to a prescribed function, namely the existence of conformal metrics $g \in\left[g_{0}\right]$ such that

$$
\sigma_{k}\left(\lambda\left(A_{g}\right)\right)=f
$$

where $f$ is a given positive smooth function on $\mathcal{M}$. As noted above, when $k>n / 2$, problem (4.21) was first solved by Gursky and Viaclovsky [30], who proved the compactness of the solution set. Their proof involved a detailed analysis of the growth rate of solutions at isolated singular points.

In [70], we prove a sharp Harnack inequality for $k$-admissible metrics when $\left(\mathcal{M}, g_{0}\right)$ is not conformally equivalent to the unit sphere $S^{n}$. As a consequence we obtained the compactness of the set of all $k$-admissible metrics with fixed volume. When $\left(\mathcal{M}, g_{0}\right)$ is the unit sphere we prove there is a unique admissible metric with singularity. As a consequence we prove an existence theorem for the equation (4.21), thereby recovering Gursky and Viaclovsky's results [30].

Our proof needs to introduce the minimal radial function and also involves nonsmooth $k$-admissible metrics, which are limit of smooth $k$-admissible functions in $L^{p}$ norm for some $p>0$. 
Minimal radial function. A main new idea in our proof is the introduction of the minimal (maximal, resp) radial function for lower (upper, resp) semicontinuous functions. For any given lower semi-continuous function $v$ in a geodesic ball $B_{R}\left(x_{0}\right) \subset \mathcal{M}$, (here $x_{0}$ can be any fixed point and $R>0$ can be any constant), the maximal radial function of $v$, also defined in $B_{R}\left(x_{0}\right)$, is a rotationally symmetric function $\tilde{v}$ satisfying $\tilde{v} \leq v$, and $\tilde{v} \geq \phi$ for any radial function $\phi$ satisfying $\phi \leq v$. Explicitly it can be given by

$$
\tilde{v}(x)=\inf \left\{v(y) \mid y \in \partial B_{r}\left(x_{0}\right), r=d\left(x, x_{0}\right)\right\} .
$$

Obviously $\tilde{v}$ is also lower semi-continuous, and for any $r \in(0, R)$, there is a point $x_{r} \in \partial B_{r}\left(x_{0}\right)$ such that $\tilde{v}\left(x_{r}\right)=v\left(x_{r}\right)$. If $v$ is superharmonic, then $\tilde{v}$ is also superharmonic, and as a function of $d\left(x, x_{0}\right)$, is monotone decreasing.

Similarly for an upper semi-continuous function $w$, the maximal radial function of $w$ is given by

$$
\tilde{w}(x)=\sup \left\{w(y) \mid y \in \partial B_{r}\left(x_{0}\right), r=d\left(x, x_{0}\right)\right\} .
$$

When the function $w$ is subharmonic (with respect to an elliptic operator), $\widetilde{w}$ is monotone increasing, and it can also be equivalently defined as follows. For any $h \in \mathbb{R}$, denote $\Omega_{h}=\{x \in \mathcal{M} \mid w(x)<h\}$, which is open as $w$ is upper semi-continuous. For any given point $x_{0}$, the minimal radial function $\widetilde{w}$ (with respect to $x_{0}$ ) can also be given by

$$
\widetilde{w}(r)=\inf \left\{h \mid \operatorname{dist}\left(x_{0}, \partial \Omega_{h}\right)>r\right\} .
$$

Note that $\widetilde{w}$ is radial, so it is a function of $r=d\left(x, x_{0}\right)$, and can be regarded as a function of one variable. Obviously $\widetilde{w} \geq w$ and $\widetilde{w} \leq \phi$ for any radial function $\phi$ satisfying $\phi \geq w$.

Nonsmooth $k$-admissible metric. Our argument involve limit of $k$-admissible metrics. Therefore we allow singular metrics and call a metric $g=\chi g_{0} k$-admissible if $\chi: \mathcal{M} \rightarrow(0, \infty], \chi$ is lower semi-continuous, $|\{x \in \mathcal{M} \mid \chi(x)=\infty\}|=0$, and there exists a sequence of $k$-admissible metrics $g_{m}=\chi_{m} g_{0}, \chi_{m} \in C^{2}(\mathcal{M})$, such that $\chi_{m} \rightarrow \chi$ almost everywhere in $\mathcal{M}$. If $g$ is $k$-admissible, then the function $v=\chi^{(n-2) / 4}$ is subharmonic with respect to the operator

$$
\square:=-\Delta_{g_{0}}+\frac{n-2}{4(n-1)} R_{g_{0}}
$$

and hence by the weak Harnack inequality [20], the set $\{\chi=\infty\}$ has measure zero.

We first prove the classification of the possible singularities of $k$-admissible metrics on $\mathbb{R}^{n}$.

THEOREM 4.2. Let $g$ be $k$-admissible metric on $\mathbb{R}^{n}$ with $\frac{n}{2}<k \leq n$. Then either

$$
g(x)=\frac{C}{\left|x-x_{0}\right|^{4}} g_{0}(x)
$$


for some point $x_{0} \in \mathbb{R}^{n}$ and positive constant $C$, or the conformal factor $\chi$ is Hölder continuous with exponent $\alpha=2-\frac{n}{k}$, where $g_{0}$ is the standard metric on $\mathbb{R}^{n}$.

Proof. Let $g=e^{-2 w} g_{0}$. Let $\widetilde{w}$ be the maximal radial function of $w$ with respect to a point $x_{0}$, which we take as the origin 0 . Then $\widetilde{w}$ is $k$-admissible in $\mathbb{R}^{n} \backslash\{0\}$. Since $\widetilde{w}$ is radial, it satisfies an ode. When $k>n / 2$, by $\sigma_{k}(\lambda(W)) \geq 0$ and from the ode we show that either $\widetilde{w}$ is Hölder continuous in $\mathbb{R}^{n}$ with exponent $\alpha=2-\frac{n}{k}$, or

$$
\widetilde{w}(x)=2 \log |x|+C
$$

for some constant C.

In the latter case, observe that the function $2 \log |x|+C$ is harmonic in $\mathbb{R}^{n} \backslash\{0\}$ and $\widetilde{w}$ is subharmonic. But by our definition of $\widetilde{w}, w-\widetilde{w}$ contains interior local maximum points. By the maximal principle, we conclude that $w(x)=2 \log |x|+C$.

By Theorem 4.2, we see that when $k>\frac{n}{2}$, there is only a unique (up to conformal diffeomorphism) singular $k$-admissible function in $\mathbb{R}^{n}$ and on $S^{n}$. This result is analogous to Obata's uniqueness result for the constant scalar curvature. But note that our uniqueness is not just for metrics with prescribed $k$-curvature, but for all $k$-admissible metrics with singularity. This result implies the set of all $k$-admissible function is quite "small". Theorem 4.2 has some other interesting consequences, see [70]. Next we prove the following Harnack inequality.

THEOREM 4.3. If $\left(\mathcal{M}, g_{0}\right)$ is not conformally equivalent to the unit sphere $S^{n}$ and $\frac{n}{2}<k \leq n$, then $\left[g_{0}\right]_{k}$ is compact in $C^{0}$ and satisfies the following Harnack inequality, namely for any $g=\chi g_{0} \in\left[g_{0}\right]_{k}$,

$$
\max _{x, y \in \mathcal{M}} \frac{\chi(x)}{\chi(y)} \leq \exp \left(C|x-y|^{2-\frac{n}{k}}\right)
$$

for some fixed constant $C$ depending only on $n, k$ and $\left(\mathcal{M}, g_{0}\right)$, where $|x-y|$ denotes the geodesic distance in the metric $g_{0}$ between $x$ and $y$.

Proof. First we note that if $u$ is $k$-admissible to the $k$-Hessian equation (1.10) and if $k>\frac{n}{2}$, then $u \in C^{\alpha}$ with $\alpha=2-\frac{n}{k}$ [67]. For $k$-admissible functions to the $k$-Yamabe problem, using the above Hölder continuity for the $k$-Hessian equation (1.10), we can prove that

$$
\frac{u(x)-u(y)}{|x-y|^{\alpha}} \leq C \int_{\mathcal{M}} u
$$

where $\alpha=2-\frac{n}{k}$ and $C$ is independent of $u$.

Next, as in the proof of Theorem 4.2, the minimal radial function of $w$ is either Hölder continuous or satisfies (4.23). In the first case, we are through. 
In the latter case, noting that the function $2 \log |x|+C$ is harmonic in $\mathbb{R}^{n} \backslash\{0\}$, and $w-\widetilde{w}$ contains interior local maximum points. By the maximal principle, a blow-up limit of $w-\widetilde{w}$ must be a constant. Hence we have

$$
w(x)=2 \log |x|+o(1) .
$$

By a similar blow-up argument we can prove the singularities are isolated. Therefore there are at most finitely many singular points.

From the singularity behavior (4.26), as in [30] we can prove that $w$ is $C^{\infty}$ smooth away from the singular points. Therefore the manifold equipped with the metric $g=e^{-2 w} g_{0}$ is a complete manifold with nonnegative Ricci curvature. By Bishop's volume comparison theorem, the quantity

$$
Q(r)=\frac{V o l_{g}\left(B_{r}(y)\right)}{r^{n}}
$$

is monotone decreasing in $r$. So $Q(r) \leq \lim _{r \rightarrow 0} Q(r)=\frac{1}{n}\left|S^{n-1}\right|$, where $\left|S^{n-1}\right|$ is the volume of the standard unit $(n-1)$-sphere. By the asymptotic estimate (4.26), we have

$$
Q(r) \rightarrow \frac{m}{n}\left|S^{n-1}\right| \quad \text { as } r \rightarrow \infty,
$$

where $m$ is the number of singular points. Therefore we have either $m=0$, or $m=1$ and $Q(r) \equiv \frac{1}{n}\left|S^{n-1}\right|$. If $m=0$, we are through. If $m=1, w$ has a unique singularity $\{0\}$. One can show that $R_{g} \equiv 0$. Hence $(\mathcal{M} \backslash\{0\}, g)$ is a complete manifold with $R i c_{g} \geq 0$. So it is isometric to the Euclidean space $\mathbb{R}^{n}$, which in turn implies that $\left(\mathcal{M}, g_{0}\right)$ is conformally equivalent to $S^{n}$. This contradicts with the assumption in Theorem 4.3.

We have thus proved that there is no singularity in $k$-admissible metrics, all conformal factors are Hölder continuous. One can furthermore prove that

$$
\begin{aligned}
& \sup _{\mathcal{M}} w-\inf _{\mathcal{M}} w \leq K, \\
& |w(x)-w(y)| \leq K|x-y|^{2-\frac{n}{k}}
\end{aligned}
$$

for some constant $K>0$. The first estimate in (4.27) can be proved by contradiction. If it is not true, there exists a sequence of $k$-admissible functions $w_{m}$ such that $\sup _{\mathcal{M}} w_{m}=0$ and $\inf _{\mathcal{M}} w_{m} \rightarrow-\infty$. Suppose that $w_{m}(0) \rightarrow-\infty$. By the Hölder continuity, we may assume that $e^{w_{m}}$ converges locally uniformly to $e^{w}$ in $\mathcal{M} \backslash\{0\}$ and $\lim _{x \rightarrow 0} w(x)=-\infty$. By a weak Harnack inequality, the set $\{w=-\infty\}$ has measure zero. So $w$ is $k$-admissible. Hence by the above argument, 0 is the unique singular point of $w, w$ is $C^{\infty}$-smooth away from 0 , and $\left(\mathcal{M}, g_{0}\right)$ is conformally equivalent to the unit sphere $S^{n}$, which contradicts with our assumption. The second estimate in (4.27) follows from (4.25).

From the Harnack inequality in Theorem 4.3 and by the Arzela-Ascoli theorem, we have the following compactness. 
Corollary 4.1. Suppose $\left(\mathcal{M}, g_{0}\right)$ is not conformally equivalent to the unit sphere $S^{n}$. Then the set of all $k$-admissible metrics of fixed volume is compact.

By the Harnack inequality in Theorem 4.3, we can not only prove the existence of solutions to equation (4.21) for positive and smooth $f$, but to more general equations. For simplicity we state the existence for a special right hand side. We refer the reader to $[\mathbf{7 0}]$ for more general existence results.

TheOrem 4.4. Let $\left(\mathcal{M}, g_{0}\right)$ be a compact $n$-manifold not conformally equivalent to the unit sphere $S^{n}$. Suppose $\frac{n}{2}<k \leq n$ and $\left[g_{0}\right]_{k} \neq \varnothing$. Then for any smooth positive function $f$ and any constant $p \neq k$, there exists a positive admissible solution to the equation

$$
\sigma_{k}(\lambda(V))=f(x) v^{p}
$$

The solution is unique if $p<k$. When $p=k$, then there exists a unique constant $\theta>0$ such that

$$
\sigma_{k}(\lambda(V))=\theta f(x) v^{k}
$$

has a solution, which is unique up to a constant multiplication.

The proof is by a degree argument as in [79]. In [79] the existence of nontrivial solution to the Monge-Ampere equation was proved for very general superlinear right hand side. The proof in [79] relies on the convexity of solutions but for equation (4.29), the Hölder continuity of solutions, implied by the Harnack inequality (4.24), is sufficient for the degree argument.

As a special case of Theorem 4.4, letting $p=k \frac{n+2}{n-2}$, we obtain the existence of the solutions to (4.21) for $n / 2<k \leq n$, which is the $k$-Yamabe problem when $f \equiv 1$, proved in $[\mathbf{3 0}]$. Note that the compactness of the set of $k$-admissible functions automatically implies that the set of solutions is compact.

4.4. The case $k=n / 2$. When $k=2$ and $n=4$, the existence and the compactness of the solution set for the $\frac{n}{2}$-Yamabe problem on compact Riemannian manifolds were proved by Chang, Gursky and Yang $([\mathbf{1 1}, \mathbf{1 2}])$. The paper $[\mathbf{1 1}]$ also contains various geometric applications of the problem. We have the following result for all even $n \geq 4[\mathbf{7 1}]$.

THEOREM 4.5. Let $\left(\mathcal{M}, g_{0}\right)$ be a compact Riemannian manifold of even dimension $n \geq 4$. Suppose $g_{0}$ is $\frac{n}{2}$-admissible and $\mathcal{M}$ is not conformally equivalent to the unit sphere. Then the set of solutions to the $\frac{n}{2}$-Yamabe problem is compact. In particular, there is a $\frac{n}{2}$-admissible solution to (4.1).

We point out that the compactness is true not only for the $k$-Yamabe problem with $k=\frac{n}{2}$, but also for the more general equation (4.21). About the proof of Theorem 4.5, we cannot prove that the maximal radial function 
$\widetilde{w}$ satisfies the asymptotic behavior (4.23) for any $k$-admissible function function $w$. But by the Liouville theorem 2.1, we can prove (4.23) if $g=e^{-2 w} g_{0}$ is a solution to the $k$-Yamabe problem. Once (4.23) is proved, by the same argument as in the case $k>\frac{n}{2}$ in $\S 4.3$, we can prove $w$ has the asymptotic behavior (4.26). Once (4.26) holds, the proof in $\S 4.3$ also applies to the case $k=\frac{n}{2}$ and we are through.

Therefore in the case $k=\frac{n}{2}$, it suffices to show that if $g=e^{-2 w} g_{0}$ is a solution to the $k$-Yamabe problem, then (4.23) holds.

The proof of (4.23) uses several results on subharmonic functions and the Liouville Theorem 2.1, which we list as follows.

(a) Let $v \geq 0$ and satisfy

$$
-\Delta v+\frac{n-2}{4(n-1)} R_{g_{0}} v \geq 0 .
$$

Then we have the weak Harnack inequality [20]

$$
\|v\|_{L^{p}(\mathcal{M})} \leq C \inf _{\mathcal{M}} v
$$

where $1 \leq p<\frac{n}{n-2}$ and $C$ is a positive constant depending on $n, p$, and $g_{0}$. (b) If $v \in B_{1}\left(x_{0}\right)$ satisfy (4.30) (in the weak sense), then $v \in W^{1, p}\left(B_{1 / 2}\right)$ for $1 \leq p<\frac{n}{n-1}$, and [32]

$$
\|v\|_{W^{1, p}\left(B_{1 / 2}\right)} \leq C\|v\|_{L^{1}\left(B_{1}\right)}
$$

where $C$ is a positive constant depending on $n, p$, and $g_{0}$.

From (4.31) and (4.32) it follows that

$$
\|v\|_{W^{1, p}(\mathcal{M})} \leq C \inf _{\mathcal{M}} v
$$

where $1 \leq p<\frac{n}{n-1}$, and $C$ depends on $n, p$, and $g_{0}$. Therefore if $v_{j}$ is a sequence of $k$-admissible functions satisfying $\inf _{\mathcal{M}} v_{j}=1$, then $v_{j}$ is uniformly bounded in $W^{1, p}(\mathcal{M})$ for any $p<\frac{n}{n-1}$, and $v_{i}$ subconverges to a (generalized) $k$-admissible function. In particular, if $v$ satisfies (4.30), then the maximal radial function $\tilde{v}$ is locally uniformly Hölder continuous away from 0 , with Hölder exponent $\alpha \in(0,1 / n)$.

(c) Let $v$ be a radially symmetric, super-harmonic function in $B_{1}(0) \subset \mathbb{R}^{n}$, namely $\Delta v \leq 0$ in $B_{1}(0)$. Then

$$
v(x)=\frac{c_{0}+o(1)}{|x|^{n-2}},
$$

where $c_{0}$ is a nonnegative constant.

(d) Suppose $\left\{v_{j}\right\}$ is a sequence of solutions with local maximum $v_{j}(0) \rightarrow \infty$. Denote

$$
w_{j}(x)=-\frac{2}{n-2} \log \frac{v_{j}(x)}{m_{j}}
$$


where $m_{j}=\inf _{\mathcal{M}} v_{j}$. Let $\widetilde{w}_{j}$ be the maximal radial function of $w_{j}$. Then $\widetilde{w}_{j} \rightarrow \widetilde{w}$ and $\widetilde{w}$ is a $k$-admissible function. By Liouville Theorem 2.1, the corresponding radial function $\widetilde{v}$ (by (4.11), $e^{\widetilde{w}}=\widetilde{v}^{-2 /(n-2)}$ ) satisfies (4.34) for some positive constant $c_{0}>0$. Hence

$$
\widetilde{w}(r) \leq 2 \log r+C .
$$

On the other hand, as $\widetilde{v}$ is $k$-admissible for some $k \geq n / 2$, it satisfies an ode and from which we have

$$
\widetilde{w}(r) \geq 2 \log r-C^{\prime}
$$

Therefore we have

$$
\widetilde{w}(r)=2 \log r+C+o(1) .
$$

namely (4.23) holds.

\section{Remarks}

5.1. Admissibility of the initial metric. In $\S 4$ we assume the initial metric $g$ is $k$-admissible, or equivalently the set $[g]_{k} \neq \emptyset$. This is a pointwise condition. For the Yamabe problem (1.6), this condition can be replaced by the Yamabe constant $Y_{1}>0$, namely if $Y_{1}>0$, then there exists a metric $\widetilde{g} \in[g]$ such that the scalar curvarture $R_{\widetilde{g}}>0$.

In the case $n=4$ and $k=2$, Chang, Gursky and Yang [11] proved that $[g]_{2} \neq \emptyset$ if the Yamabe constants $Y_{1}>0$ and $Y_{2}>0$. See also [29] for a different proof. The Yamabe constants $Y_{1}$ and $Y_{2}$ are defined as follows.

$$
\begin{aligned}
& Y_{1}=\inf _{\widetilde{g} \in[g]} \operatorname{vol}(\widetilde{g})^{-\frac{n-2}{n}} \int_{\mathcal{M}} \sigma_{1}(\widetilde{g}) d v o l_{\widetilde{g}} \\
& Y_{2}=\inf _{\widetilde{g} \in[g]} \operatorname{vol}(\widetilde{g})^{-\frac{n-4}{n}} \int_{\mathcal{M}} \sigma_{2}\left(\widetilde{g}^{-1} A_{\widetilde{g}}\right) d \operatorname{vol}_{\widetilde{g}} .
\end{aligned}
$$

By Chern-Gauss-Bonnet Formula, $Y_{2}$ is a conformal invariant when $n=4$.

Denote

$$
\hat{Y}_{k}([g])= \begin{cases}\inf \left\{\mathcal{F}_{k}(\widetilde{g}) \mid \widetilde{g} \in[g]_{k-1}, \operatorname{vol}(\mathcal{M}, \widetilde{g})=1\right\} & \text { if }[g]_{k-1} \neq \emptyset \\ -\infty, & \text { if }[g]_{k-1}=\emptyset\end{cases}
$$

where $\mathcal{F}_{k}$ is given in (4.4). Then we have

THEOREM 5.1. Let $(\mathcal{M}, g)$ be a compact $n$-dimensional manifold. Assume that $\hat{Y}_{k}([g])>0$ for $k \geq 2$. Then there exists a $k$-admissible conformal metric $\widetilde{g} \in[g]$.

This result was proved by the first author in [59]. For locally conformally flat manifolds, it was proved by Guan, Lin and Wang $[\mathbf{2 3}]$. 
Proof. Consider a conformal metric $g=e^{-2 u} g_{0}$ satisfying

$$
\sigma_{k}^{1 / k}\left(g_{0}^{-1} A_{g}^{t}\right)=f(x) e^{2 u},
$$

where

$$
A^{t}=\frac{1}{n-2}\left(\operatorname{Ric}_{g}-\frac{t}{2(n-1)} R_{g} \cdot g\right)
$$

$t \leq 1$, and $f(x)>0$ is any smooth function. The idea of introducing the family of equations is such that the equation becomes uniformly elliptic when $t<1$. This idea was used by Krylov [37] and Trudinger [64]. One may easily show that the linearized operator of this equation is invertible. Choosing a proper function $f$, we can establish the a priori estimates for solutions of (5.2), and by the continuity method, there exists a solution of (5.2) for $t=1$.

5.2. Existence of solutions for more general nonlinear curvature functions. In the case when $\mathcal{M}$ is locally conformally flat, as explained in $\S 4.1$, the proof is based on the a priori estimates and the moving plane argument. Therefore it applies to more general nonlinear curvatures. Similarly the compactness results in $\S 4.3$ and 4.4 relies essentially on the condition $k \geq \frac{n}{2}$, and they can also be extended to more general curvature functions. More specifically, these results can be extended to the following more general equation

$$
\sigma\left(\lambda\left(A_{\tilde{g}}\right)\right)=\phi
$$

where $\phi$ is a positive, smooth function on $\mathcal{M}, \sigma$ is a nonlinear function defined on an open convex cone $\Gamma \subset \mathbb{R}^{n}$. We assume that $\sigma$ satisfies

$\left(\mathbb{C}_{1}\right) \sigma>0$ in $\Gamma$ and $\sigma=0$ on $\partial \Gamma$;

$\left(\mathbb{C}_{2}\right) \sigma$ is concave;

$\left(\mathbb{C}_{3}\right) \sigma$ is invariant under exchange of variables;

$\left(\mathbb{C}_{4}\right) \sigma$ is homogeneous of degree 1.

We also assume that $\Gamma$ satisfies

$\left(\mathbb{G}_{1}\right)$ If $\lambda \in \Gamma$, then any permutation of $\lambda$ also lies in $\Gamma$;

$\left(\mathbb{G}_{2}\right) \Gamma_{n} \subset \Gamma \subset \Sigma_{\frac{1}{n-2}}$, where $\Gamma_{n}$ is the positive cone and

$$
\Sigma_{\theta}=\left\{\lambda \in \mathbb{R}^{n} \mid P_{\theta}(\lambda)>0\right\},
$$

and $P_{\theta}$ is the Pucci operator given after Proposition 4.2.

Then the existence results on locally conformally flat manifolds in $\S 4.1$ can be extended to equation (5.3) if $\mathbb{C}_{1}-\mathbb{C}_{4}$ and $\mathbb{G}_{1}$ hold; and the existence and compactness results in Theorem 4.5 holds in $\mathbb{C}_{1}-\mathbb{C}_{4}$ and $\mathbb{G}_{1}-\mathbb{G}_{2}$ hold.

Recently there have also been some interesting works on the singularity analysis for $k$-admissible functions and on the a priori estimates and existence of solutions for the $k$-Yamabe problem on manifolds with boundary. However, research in these directions are in progress and we will not discuss these aspects in this work. 
5.3. Open problems. We finish this paper by mentioning a few unsolved problems.

1. A natural question is the existence of solutions to the $k$-Yamabe problem when $2<k<n / 2$ and $\mathcal{M}$ is not locally conformally flat.

2 . The compactness of the solution set to the $k$-Yamabe problem for $k=1$ and $k \geq \frac{n}{2}$ has been completely solved. One would also like to know the answer for the cases $2 \leq k<\frac{n}{2}$, in particular the case $k=2$.

3 . The full $k$-Yamabe problem $[\mathbf{3 8}]$. In the introduction of the $k$-Yamabe problem we have dropped the Weyl tensor in the decomposition Riem $=W+A_{g} \odot g$. Labbi $[\mathbf{3 8}]$ proposed to study the existence of a conformal metric such that the quantity $s_{k}:=c^{2 k} \mathrm{Riem}^{k}$ is equal to a constant, where $c$ is the standard contraction operator, and Riem $^{k}$ denotes the Kulkarni-Nomizu product. When $k=1, s_{k}$ is exactly the scalar curvature of $(\mathcal{M}, g)$. When $k=\frac{n}{2}$ and $n$ is even, $s_{k}$ is the Lipschitz-Killing curvature. As shown by Labbi, this quantity $s_{k}$ is always variational and if $\mathcal{M}$ is a hypersurface, $s_{k}$ is the $(2 k)^{t h}$ mean curvature of $\mathcal{M}$, namely the $(2 k)^{t h}$ elementary symmetric polynomial of the principal curvatures of $\mathcal{M}$. When $\mathrm{M}$ is locally conformally flat, then the Weyl curvature vanishes, and $s_{k}=\sigma_{k}\left(\lambda\left(A_{g}\right)\right)$ for $k=1,2, \ldots,\left[\frac{n}{2}\right]$ (up to a constant).

\section{References}

[1] T. Aubin, Équations différentielles non linéaires et problème de Yamabe concernant la courbure scalaire, J. Math. Pures Appl. 55 (1976), 269-296.

[2] T. Aubin, Problèmes isopérimetriques et espaces de Sobolev, J. Diff. Geom. 11 (1976), $573-598$.

[3] T. Aubin, Some nonlinear problems in Riemannian geometry, Springer, 1998.

[4] A.L. Besse, Einstein manifolds, Springer-Verlag, Berlin, 1987.

[5] T. Branson and R. Gover, Variational status of a class of fully nonlinear curvature prescription problems, Calc. Var. PDEs 32 (2008), 253-262.

[6] S. Brendle, Blow-up phenomena for the Yamabe PDE in high dimensions, J. Amer. Math. Soc. 21 (2008), 951-979.

[7] S. Brendle and F. C. Marques, Blow-up phenomena for the Yamabe problem II, J. Diff. Geom. 81(2009), 225-250.

[8] S. Brendle and J. Viaclovsky, A variational characterization for $\sigma_{n / 2}$, Calc. Var. PDEs 20(2004), 399-402.

[9] L.A. Caffarelli, B. Gidas and J. Spruck, Asymptotic symmetry and local behavior of semilinear elliptic equations with critical Sobolev growth, Comm. Pure Appl. Math., 42 (1989), 271-297.

[10] L.A. Caffarelli, L. Nirenberg, and J. Spruck, Dirichlet problem for nonlinear second order elliptic equations III, Functions of the eigenvalues of the Hessian, Acta Math. 155(1985), 261-301.

[11] A. Chang, M. Gursky, P. Yang, An equation of Monge-Amère type in conformal geometry, and four-manifolds of positive Ricci curvature, Ann. of Math. (2) 155(2002), 709-787.

[12] A. Chang, M. Gursky, P. Yang, An a priori estimate for a fully nonlinear equation on four-manifolds, J. Anal. Math. 87 (2002), 151-186. 
[13] A. Chang, M. Gursky, and P. Yang, Entire solutions of a fully nonlinear equation, Lectures on partial differential equations, 43-60, Inter. Press, 2002.

[14] S. Chen, Local estimates for some fully nonlinear elliptic equations, Int. Math. Res. Not., 55 (2005), 3403-3425.

[15] K.S. Chou (K. Tso), On a real Monge-Amperé functional, Invent. Math. 101(1990), 425-448.

[16] K.S. Chou and X.-J. Wang, A variational theory of the Hessian equation, Comm. Pure Appl. Math. 54 (2001), 1029-1064.

[17] O. Druet, Compactness for Yamabe metrics in low dimensions, Int. Math. Res. Not., 23 (2004), 1143-1191.

[18] L. C. Evans, Classical solutions of fully nonlinear, convex, second-order elliptic equations, Comm. Pure Appl. Math., 35 (1982), 333-363.

[19] B. Gidas, W. M. Ni and L. Nirenberg, Symmetry and related properties via the maximum principle, Comm. Math. Phys., 68(1979), 209-243.

[20] D. Gilbarg and N.S. Trudinger, Elliptic partial differential equations of second order, Springer, Berlin, 1983.

[21] B. Guan, The Dirichlet problem for Hessian equations on Riemannian manifolds, Calc. Var. PDEs, 8 (1999), 45-69.

[22] B. Guan, Conformal metrics with prescribed curvature functions on manifolds with boundary, Amer. J. Math. 129 (2007), 915-942.

[23] P. Guan, C. S. Lin and G. Wang, Application of the method of moving planes to conformally invariant equations, Math. Z. 247 (2004), 1-19.

[24] P. Guan, J. Viaclovsky and G. Wang, Some properties of the Schouten tensor and applications to conformal geometry, Trans. Amer. Math. Soc. 355 (2003), 925-933.

[25] P. Guan and G. Wang, Local estimates for a class of fully nonlinear equations arising from conformal geometry, Int. Math. Res. Not. (2003), 1413-1432.

[26] P. Guan and G. Wang, A fully nonlinear conformal flow on locally conformally flat manifolds, J. Reine Angew. Math. 557 (2003), 219-238.

[27] P. Guan and G. Wang, Geometric inequalities on locally conformally flat manifolds, Duke Math. J. 124 (2004), 177-212.

[28] P. Guan and X.-J. Wang, On a Monge-Ampère equation arising in geometric optics, J. Diff. Geom., 48(1998), 205-223.

[29] M. Gursky and J. Viaclovsky, A fully nonlinear equation on four-manifolds with positive scalar curvature, J. Differential Geom. 63 (2003), 131-154.

[30] M. Gursky and J.Viaclovsky, Prescribing symmetric functions of the eigenvalues of the Ricci tensor, Ann. of Math. (2) 166 (2007), 475-531.

[31] F.R. Harvey and H.B. Lawson, Dirichlet duality and the nonlinear Dirichlet problem, Comm. Pure Appl. Math. 62 (2009), 396-443.

[32] J. Heinonen, T. Kilpelainen, O. Martio, Nonlinear potential theory of degenerate elliptic equations, Oxford Univ. Press, 1993.

[33] N. Ivochkina, Solution of the Dirichlet problem for certain equations of MongeAmpère type (Russian), Mat. Sb. (N.S.) 128 (1985), 403-415.

[34] M.A. Khuri, F.C. Marques and R. M. Schoen, A compactness theorem for the Yamabe problem, J. Diff. Geom. 81 (2009), 143-196.

[35] N. Korevaar, R. Mazzeo,F. Pacard, R. Schoen, Refined asymptotics for constant scalar curvature metrics with isolated singularities, Invent. Math. 135 (1999), 233-272.

[36] N. V. Krylov, Boundedly inhomogeneous elliptic and parabolic equations in a domain, Izv. Akad. Nauk SSSR Ser. Mat., 47 (1983), 75-108.

[37] N.V. Krylov, Nonlinear elliptic and parabolic equations of second order, Reidel, 1987.

[38] M.-L. Labbi, Variational properties of the Gauss-Bonnet curvatures, Calc. Var. PDEs 32 (2008), 175-189.

[39] D. Labutin, Potential estimates for a class of fully nonlinear elliptic equations, Duke Math. J. 111 (2002), 1-49. 
[40] J.M. Lee and T.H. Parker, The Yamabe problem, Bull. Amer. Math. Soc. 17(1987), $37-91$.

[41] A. Li and Y.Y. Li, On some conformally invariant fully nonlinear equations, Comm. Pure Appl. Math. 56 (2003), 1416-1464.

[42] A. Li and Y.Y. Li, On some conformally invariant fully nonlinear equations II, Liouville, Harnack, and Yamabe, Acta Math. 195 (2005), 117-154.

[43] Y.Y. Li, Degenerate conformally invariant fully nonlinear elliptic equations, Arch. Ration. Mech. Anal. 186 (2007), 25-51.

[44] Y.Y. Li, Local gradient estimates of solutions to some conformally invariant fully nonlinear equations, C. R. Math. Acad. Sci. Paris 343 (2006), 249-252; Comm. Pure Appl. Math. 62 (2009), 1293-1326.

[45] Y.Y. Li and L. Zhang, Compactness of solutions to the Yamabe problem II, Calc. Var. PDE, 24 (2005), 185-237.

[46] Y.Y. Li and L. Zhang, Compactness of solutions to the Yamabe problem III ,J. Funct. Anal., 245 (2007), 438-474.

[47] Y.Y. Li and M. Zhu, Yamabe type equations on three-dimensional Riemannian manifolds, Commun. Contemp. Math. 1 (1999), 1-50.

[48] X.N. Ma, N.S. Trudinger, and X.-J. Wang, Regularity of potential functions to the optimal transportation problem, Arch. Ration. Mech. Anal. 177 (2005), 151-183.

[49] F.C. Marques, A priori estimates for the Yamabe problem in non-locally conformal flat cases, J. Diff. Geom. 71 (2005), 315-346.

[50] M. Obata, the conjecture on conformal transformations of Riemannian manifolds, J. Diff. Geom. 6 (1971), 247-258.

[51] R. Schoen, Conformal deformation of a Riemannian metric to constant scalar curvature, J. Diff. Geom. 20(1984), 479-495.

[52] R. Schoen, Variational theory for the total scalar curvature functional for Riemannian metrics and related topics. Topics in Calculus of Variations, Lectures Notes in Math. 1365 (1989), pp. 120-154.

[53] R. Schoen, Lecture notes on differential geometry, 1988 at Stanford Univ, 1989 at Courant Inst.

[54] R. Schoen, On the number of constant scalar curvature metrics in a conformal class, Differential geometry, 311-320, Pitman Monogr. Surveys Pure Appl. Math. 52, 1991.

[55] R. Schoen and S.T. Yau, Conformally flat manifolds, Kleinian groups and scalar curvature, Invent. Math. 92 (1988), 47-71.

[56] R. Schoen and S.T. Yau, Lectures on Differential geometry, International Press, 1994.

[57] R. Schoen and D. Zhang, Prescribed scalar curvature on the $n$-sphere, Calc. Var. PDEs 4(1996), 1-25.

[58] F. Schulz, Regularity theory for quasi-linear elliptic systems and Monge-Ampère equations in two dimensions, Lecture Notes in Math. vol. 1445, Springer, 1990.

[59] W.M. Sheng, Admissible metrics in the $\sigma_{k}$ Yamabe problem, Proc. Amer. Math. Soc. 136 (2008), 1795-1802.

[60] W.M. Sheng, N.S. Trudinger and X.-J. Wang, The Yamabe problem for higher order curvatures, J. Diff. Geom. 77(2007), 515-553.

[61] G. Tian and X.-J. Wang, Moser-Trudinger type inequalities for the Hessian equation, J. Funct. Anal., 259 (2010), 1974-2002.

[62] G. Tian and X.-J. Wang, A priori estimates for fully nonlinear parabolic equations, preprint, 2011.

[63] N.S. Trudinger, Remarks concerning the conformal deformation of Riemannian structures on compact manifolds, Ann. Scuola Norm. Sup. Pisa (3) 22(1968), 265-274.

[64] N.S. Trudinger, The Dirichlet problem for the prescribed curvature equations, Arch. Rat. Mech. Anal. 111 (1990), 153-179.

[65] N.S. Trudinger, On the Dirichlet problem for Hessian equations, Acta Math., 175(1995), 151-664. 
[66] N.S. Trudinger and X.-J. Wang, Hessian measures I, Topol. Methods Nonlinear Anal. 10 (1997), 225-239.

[67] N.S. Trudinger and X.-J. Wang, Hessian measures II, Ann. of Math. (2) 150 (1999), 579-604.

[68] N.S. Trudinger and X.-J. Wang, Hessian measures III, J. Funct. Anal. 193 (2002), $1-23$.

[69] N.S. Trudinger and X.-J. Wang, A Poincaré type inequality for Hessian integrals, Calc. Var. PDEs 6(1998), 315-328.

[70] N.S. Trudinger and X.-J. Wang, On Harnack inequalities and singularities of admissible metrics in the Yamabe problem, Calc. Var. PDEs 35 (2009), 317-338.

[71] N.S. Trudinger and X.-J. Wang, The intermediate case of the Yamabe problem for higher order curvatures, Int. Math. Res. Not. Vol. 2010, No. 13, pp. 2437-2458.

[72] J. Viaclovsky, Conformal geometry, contact geometry, and the calculus of variations, Duke Math. J. 101 (2000), 283-316.

[73] J. Viaclovsky, Estimates and existence results for some fully nonlinear elliptic equations on Riemannian manifolds, Comm. Anal. Geom. 10 (2002), 815-846.

[74] J. Viaclovsky, Conformally invariant Monge-Ampere equations: global solutions, Trans. Amer. Math. Soc., 352 (2000), 4371-4379.

[75] X.-J. Wang, A class of fully nonlinear elliptic equations and related functionals, Indiana Univ. Math. J. 43 (1994), 25-54.

[76] X.-J. Wang, On the design of a reflector antenna, Inverse Problems, 12(1996), 351375.

[77] X.-J. Wang, The $k$-Hessian equation, Geometric analysis and PDEs, 177-252. Lecture Notes in Math., vol.1977, Springer, 2009.

[78] X.-J. Wang, A priori estimates and existence for a class of fully nonlinear elliptic equations in conformal geometry, Chinese Ann. Math. Ser. B 27 (2006), 169-178.

[79] X.-J. Wang, Existence of multiple solutions to the equations of Monge-Ampère type, J. Differ. Eqs., 100 (1992), 95-118.

[80] H. Yamabe, On a deformation of Riemannian structures on compact manifolds, Osaka Math. J. 12(1960), 21-37.

[81] R. Ye, Global existence and convergence of Yamabe flow, J. Diff. Geom. 39 (1994), $35-50$.

Centre for Mathematics and Its Applications, The Australian National University, Canberra, ACT 0200, Australia; And, Department of Mathematics, Zhejiang University, Hangzhou, 310027, P.R. China.

E-mail address: weimins@zju.edu.cn

Centre for Mathematics and Its Applications, The Australian National University, CANBERRA, ACT 0200,

Australia.

E-mail address: Neil.Trudinger@anu.edu.au, Xu-Jia.Wang@anu.edu.au 
